\title{
The importance of applied light quality on the production of lignans and phenolic acids in Schisandra chinensis (Turcz.) Baill. cultures in vitro
}

\author{
Agnieszka Szopa $^{1} \cdot$ Halina Ekiert ${ }^{1}$
}

Received: 21 March 2016/Accepted: 22 June 2016/Published online: 28 June 2016

(c) The Author(s) 2016. This article is published with open access at Springerlink.com

\begin{abstract}
Shoot-differentiating callus cultures of Schisandra chinensis were cultivated under different spectral properties of monochromatic light (far-red, red, blue, UVA), and in darkness and under white light (control conditions). The applied lighting conditions influenced biomass growth and the accumulation of secondary metabolitesdibenzocyclooctadiene lignans (fourteen compounds) and phenolic acids (seven compounds). Total amounts of the estimated metabolites increased 1.71 and 1.98 times, respectively, depending on light quality. Blue light was found to be the most effective lighting to the production of both groups of compounds. Their total amounts reached the maximum values of $376.41 \mathrm{mg} / 100 \mathrm{~g} \mathrm{DW}$ and $46.57 \mathrm{mg} /$ $100 \mathrm{~g} \mathrm{DW}$, and were correspondingly 1.31 and 1.37 times greater than under white light. The amounts of individual compounds from the tested groups increased from 1.51 to 3.38 times (lignans), and from 1.74 to 2.72 times (phenolic acids), depending on the lighting conditions. The main bioactive compounds obtained in high amounts were: schisandrin $(67.70 \mathrm{mg} / 100 \mathrm{~g}$ DW), deoxyschisandrin (55.19 mg/100 g DW), gomisin A (36.97 mg/100 g DW), and also chlorogenic acid (15.33 mg/100 g DW) and protocatechuic acid $(13.11 \mathrm{mg} / 100 \mathrm{~g} \mathrm{DW})$. This is the first report providing evidence of the importance of light quality on the production of dibenzocyclooctadiene lignans and phenolic acids in the biomass of $S$. chinensis cultivated in vitro.
\end{abstract}

Agnieszka Szopa

a.szopa@uj.edu.pl

1 Chair and Department of Pharmaceutical Botany, Jagiellonian University, Collegium Medicum, ul. Medyczna 9, 30-688 Kraków, Poland
Keywords Schizandra - Chinese magnolia vine . Dibenzocyclooctadiene lignans $\cdot$ Schisandrin · Gomisin A . Deoxyschisandrin

$\begin{array}{ll}\text { Abbreviations } \\ \text { BA } & \text { 6-Benzyladenine } \\ \text { DW } & \text { Dry weight } \\ \text { HPLC } & \text { High-performance liquid chromatography } \\ \text { MS } & \text { Murashige and Skoog } \\ \text { NAA } & \text { 1-Naphthaleneacetic acid }\end{array}$

\section{Introduction}

Schisandra chinensis (Turcz.) Baill.- the Chinese magnolia vine (Schisandraceae), is a valuable East-Asian medicinal plant species (Szopa and Ekiert 2014; Szopa et al. 2016a). S. chinensis has long been known in Far Eastern medicine, and since 2008 has been made known and utilized in the authorative European therapy as a pharmacopoeial plant species (European Directorate for the Quality of Medicines 2008). This species is also listed in the United States Pharmacopeia (1999), as well as in the International Pharmacopoeia published by WHO (World Health Organization 2007).

The plant raw material- the Schisandra fruit, shows valuable biological activities, such as hepatoregenerative, adaptogenic, antioxidant, antiphlogistic and antitumor properties (Hancke et al. 1999; Szopa et al. 2016a). The responsibility for all these therapeutic properties is attributed to dibenzocyclooctadiene lignans, referred to in the scientific literature as schisandra lignans (World Health Organization 2007). Recently, we documented considerable production of these compounds in the biomass of high-degree organogenesis-in shoot-differentiating callus 
cultures of this species cultivated in different in vitro systems (Szopa and Ekiert 2011, 2013, 2015; Szopa et al. 2015, 2016b).

The schisandra lignans have a distinctive chemical structure and their biogenesis has not yet been explored in detail, although the initial steps of this process are known to belong to the shikimic acid pathway, which is also involved in the biogenesis of phenolic acids. Phenolic acids, numerous cinnamic or benzoic acid derivatives and depsides, such as chlorogenic and rosmarinic acids, exhibit cholagogic, spasmolytic, anti-inflammatory, anticancer, immunostimulating, antioxidant and anti-radical attributes (Chlopčikova et al. 2004; Khadem and Marles 2010). These features had inspired us to investigate the accumulation of this group of plant secondary metabolites also in cultures of $S$. chinensis in vitro. Those studies were the first of their kind. For the first time, we had documented the ability of two types of in vitro $S$. chinensis cultures (shootdifferentiating callus culture and undifferentiating callus culture) to produce some free phenolic acids (Szopa and Ekiert 2012).

Bioprocess development and scale-up of in vitro cultures allow the control of the physical and chemical parameters for the production of cell mass and secondary metabolites. In our previous studies, we have tested the effect of concentrations of some plant growth regulatorsBA (6-benzyladenine) and NAA (1-naphthaleneacetic acid) on $S$. chinensis biomass and secondary metabolites production in different in vitro systems - agar and liquid systems (Szopa et al. 2016b).

One of the most important physical factors for the increase in cell biomass and storing of secondary metabolites are lighting conditions. This includes light intensity and photoperiod. In the case of monochromatic light, the wavelength is also of significance (Ramawat and Mathur 2007). The favourable effect of light quality on the accumulation of different groups of compounds, such as flavonoids (Krewzaler and Hahlbrock, 1973), anthocyanins (Zhong et al. 1991), betalains (Shin et al. 2004) and ginsenosides ( $\mathrm{Yu}$ et al. 2005), has been shown. Also our team had documented the significance of lighting conditions on the production yields of phenolic acids and linear furanocoumarins in Ruta graveolens and $R$. g. ssp. divaricata cultures in vitro (Szopa et al. 2012).

The purpose of the present study was to investigate the significance of light with different wavelengths: far-red, red, blue, and UV-A, as well as darkness and white light (as the control conditions), for the accumulation of dibenzocyclooctadiene lignans and free phenolic acids, including cinnamic acid-one of the parent compounds of phenolic acids in the biomass from $S$. chinensis shoot-differentiating callus culture. The ultimate objective was to propose lighting conditions for increased accumulation of both groups of these bioactive metabolites. The in vitro cultures were maintained on Murashige and Skoog (MS 1962) medium supplemented with $3.0 \mathrm{mg} / 1$ of $\mathrm{BA}$ and $1.0 \mathrm{mg} / \mathrm{l}$ of NAA. This combination of plant growth regulators had proved to be an excellent, universal 'growth' and 'production' medium in our previous studies on agar shootdifferentiating callus cultures of S. chinensis (Szopa and Ekiert 2011, 2013, 2015; Szopa et al. 2016b).

The identification of all dibenzocyclooctadiene lignans was performed by LC-DAD-ESI-MS and DAD-HPLC analyses. Quantification of nine lignans was performed based on the DAD-HPLC method. The quantity of five tentatively identified lignans (based only on LC-DAD-ESIMS analyses), for which standards are not commercially available, was determined with the help of a calibration curve for schisandrin as the major component of the group of dibenzocyclooctadiene lignans (Szopa et al. 2016b; Zhang et al. 2009). The quantitative analyses of free phenolic acids and cinnamic acid were carried out by the DAD-HPLC method (Ellnain-Wojtaszek and Zgórka 1999).

This present study is unique to investigate the effect of light quality on the accumulation of dibenzocyclooctadiene lignans and phenolic acids in in vitro cultures of $S$. chinensis.

\section{Materials and methods}

\section{Experimental in vitro cultures}

The shoot-differentiating callus cultures of Schisandra chinensis were established and cultivated as was described previously (Szopa and Ekiert 2011). Experimental agar shoot-differentiating callus cultures (three series) were maintained on the MS medium (1962) supplemented with $3.0 \mathrm{mg} / \mathrm{l}$ of cytokinin-BA and $1.0 \mathrm{mg} / \mathrm{l}$ of auxin-NAA. Cultures were maintained under different lighting conditions at a temp. of $25 \pm 2{ }^{\circ} \mathrm{C}$, in vessels for plant tissue cultures $(100 \mathrm{ml}, 66 \mathrm{~mm} \times 59 \mathrm{~mm}$, product no. V8630, Sigma-Aldrich Co.) with special Magenta B-caps (product no. B8648, Sigma-Aldrich Co.). Inoculum (1.0 g) was transferred into the vessel containing $25 \mathrm{ml}$ of an agarsolidified medium at first day of experiments. Cultures were collected after 30 days of growth.

\section{Lighting conditions}

The cultures were cultivated under continuous light of various spectra: far-red light $(770-800 \mathrm{~nm}$, incandescent light and filters: standard filter no. 405 orange + standard filter no. 420 deep blue, Compact light B.V. Amsterdam, $100 \mathrm{~W})$, red (647-770 nm, Philips TLD, $36 \mathrm{~W}$ lamp), blue 
light (450-492 nm, Philips TLD, $36 \mathrm{~W}$ lamp), and UV-A irradiation (360-450 nm, Philips TLD $36 \mathrm{~W}$ lamp), and in darkness during the course of the whole experiment. Additionally, the control cultures were maintained under continuous white light (390-760 nm, Tungsram F33, 40 W lamp). Radiometric readings were made on a horizontal surface $35 \mathrm{~cm}$ above the cultures with a spectroradiometer (LI-1800, LI-COR USA). The photosynthetically active radiation was $60 \mu \mathrm{mol} \mathrm{m} \mathrm{m}^{-2} \mathrm{~s}^{-1}$, but for the red and far-red lights it was only $20 \mu \mathrm{mol} \mathrm{m} \mathrm{m}^{-2} \mathrm{~s}^{-1}$.

\section{Analysis of dibenzocyclooctadiene lignans}

Lyophilized biomass $(0.5 \mathrm{~g})$ harvested at the end of 30-day cycles (three series) of growth under different lighting conditions and darkness, and under white light as the control, was extracted by ultrasonication (twice with $50 \mathrm{ml}$ of methanol for $30 \mathrm{~min}$ ). In the extracts, chromatographic quantification of lignans was performed by the HPLCDAD method (Zhang et al. 2009) using a Kinetex ${ }^{\mathrm{TM}} \mathrm{C}-18$ analytical column $(150 \times 4.6 \mathrm{~mm}, 2.6 \mu \mathrm{m}$, Phenomenex $)$. The mobile phase consisted of acetonitrile (A) and water (B) with gradient elution (B from 40 to $95 \%$ ). Injection volume was $5 \mu \mathrm{l}$. Detection was measured at wavelength $\lambda=225 \mathrm{~nm}$. The analysis was performed at temp. $30^{\circ} \mathrm{C}$. Identification and quantification were made by comparison with nine standards: schisandrin $\mathrm{C}$, schisantherin $\mathrm{B}$ and schisanthenol (ChemFaces Biochemical), gomisin A, deoxyschisandrin, schisandrin and $\gamma$-schisandrin (ChromaDex), gomisin G and schisantherin A (PhytoLab). Quantification of the provisionally determined compounds for which standards are not commercially available (based on LC-DAD-ESI-MS analyses, described previously, acc. to Szopa et al. 2016b) was performed using a calibration curve for schisandrin as the main component of the group of dibenzocyclooctadiene lignans. The tentatively identified compounds were: angeloyl-/tigloylgomisin $\mathrm{H}$, angeloyl-/tigloylgomisin Q, benzoylgomisin P, schisandrin B and schisantherin D (or one of its stereoisomers-benzoylgomisin $\mathrm{O}$ or benzoylisogomisin $\mathrm{O}$ ).

\section{Analysis of phenolic acids}

Samples of lyophilized biomass from the in vitro cultures (0.5 g each), collected after the 30-day cycles (three series) of growth under different lighting conditions including white light as the control, were extracted twice with boiling methanol (50 $\mathrm{ml}$ for $2 \mathrm{~h}$ using a reflux condenser).

Estimation of free phenolic acids was performed using a modified HPLC-DAD method (Ellnain-Wojtaszek and Zgórka, 1999). An HPLC-DAD apparatus (Merck-Hitachi) and a Purospher RP-18e column $(4 \times 250 \mathrm{~mm}, 5 \mu \mathrm{m}$; Merck) were used. The mobile phase consisted of: A- methanol: $0.5 \%$ acetic acid $(1: 4 \mathrm{v} / \mathrm{v})$; $\mathrm{B}-$ methanol, at gradient program (B from 0 to $100 \%$ ), at temp. $25^{\circ} \mathrm{C}$. The flow rate was $1 \mathrm{ml} / \mathrm{min}$., injection volume was $10 \mu \mathrm{l}$, and detection wavelength was $254 \mathrm{~nm}$. Quantification was carried out based on the calibration curves for the standards of the following phenolic acids: 3,4-dihydroxyphenylacetic, caffeic, chlorogenic, $o$-coumaric, $m$-coumaric, $p$ coumaric, ferulic, gallic, gentisic, hydrocaffeic, $p$-hydroxybenzoic, isoferulic, neochlorogenic, protocatechuic, rosmarinic, salicylic, sinapic, syringic, vanillic acids, and also of cinnamic acid (Sigma-Aldrich Co.).

\section{Statistical analysis}

Results were presented as mean \pm standard deviation (SD) and were compared by the one-way analysis of variance (one-way ANOVA). For the comparison between different groups post hoc Tukey HSD (honestly significant difference) was used. The $p$ value $<0.05$ was considered statistically significant. STATISTICA version 12 PL software package (StatSoft) was used for the analysis.

\section{Results}

\section{Biomass increments}

The influence of the different light spectra on biomass growth in shoot-differentiating callus cultures of Schisandra chinensis was noticeable. The increases in dry biomass varied from 2.02- to 4.42-fold within the 30-day growth cycles (Fig. 1). The best growth, where the most vigorous, dark-green shoot-differentiating callus obtained, was blue light (maximum increase in dry biomass). In the control (under white light), the obtained biomass increments were equal to 3.83 .

\section{Production of dibenzocyclooctadiene lignans}

In biomass extracts from all the examined variants of monochromatic light, all of the fourteen dibenzocyclooctadiene lignans were analyzed qualitatively and quantitatively. The total amounts of lignans were dependent on light quality and ranged from $220.48 \mathrm{mg} / 100 \mathrm{~g}$ DW (UV-A irradiation) to $376.41 \mathrm{mg} / 100 \mathrm{~g} \mathrm{DW}$ (blue light) (Table 1). Apart from the blue light, another lighting condition that fostered the accumulation of schisandra lignans was illumination with the far-red light - the total amount of lignans was $306.32 \mathrm{mg} / 100 \mathrm{~g} \mathrm{DW}$. The total amounts of lignans in extracts from the biomass cultivated under the other lighting conditions were lower-262.85 mg/100 g DW in red light and $237.69 \mathrm{mg} / 100 \mathrm{~g} \mathrm{DW}$ in darkness. In the control (white light), the total amount was $286.62 \mathrm{mg} / 100 \mathrm{~g} \mathrm{DW}$. 


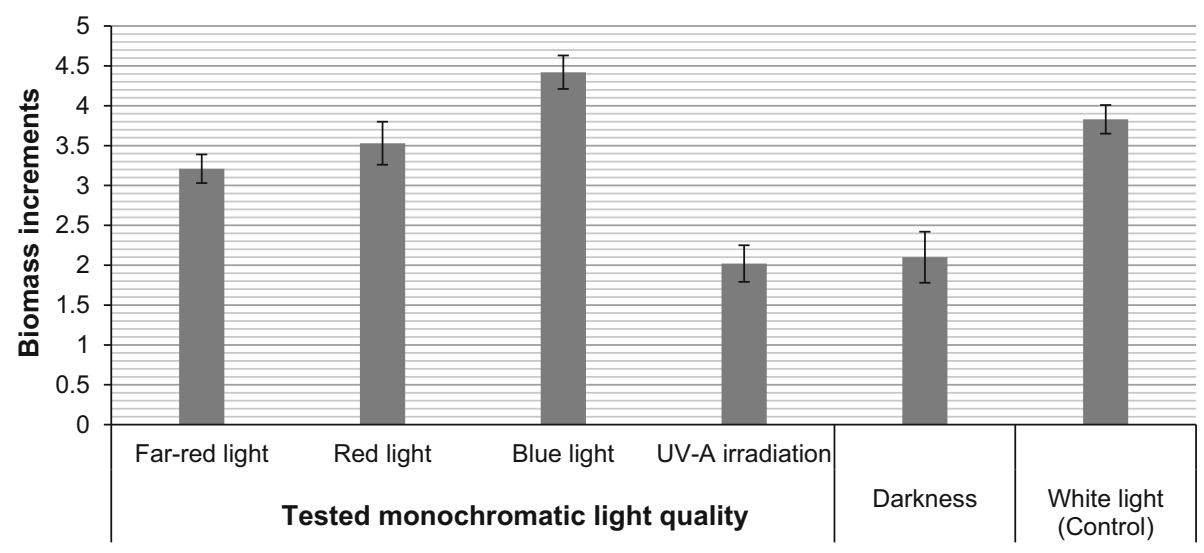

Fig. 1 Increments $\left(\frac{D W 1-D \text { Winoculum }}{D \text { Winoculum }}\right) \pm \mathrm{SD}$ of dry biomass of shoot-differentiating callus cultures of Schisandra chinensis cultivated under different light conditions

The quantities of particular compounds ranged from $0.71 \mathrm{mg} / 100 \mathrm{~g}$ DW to $69.12 \mathrm{mg} / 100 \mathrm{~g}$ DW. The predominant compounds were: schisandrin (max. $67.70 \mathrm{mg} / 100 \mathrm{~g}$ DW), deoxyschisandrin (max. $55.19 \mathrm{mg} / 100 \mathrm{~g} \mathrm{DW}$ ) and gomisin A (max. $36.97 \mathrm{mg} / 100 \mathrm{~g} \mathrm{DW})$. There were also considerable quantities of the tentatively recognized lignans: angeloyl-/tigloylgomisin Q (max. $69.12 \mathrm{mg} / 100 \mathrm{~g}$ DW), angeoyl-/tigloylgomisin $\mathrm{H}(\max .39 .95 \mathrm{mg} / 100 \mathrm{~g}$ DW), and benzoylgomisin P (max. $35.57 \mathrm{mg} / 100 \mathrm{~g} \mathrm{DW}$ ).

\section{Production of phenolic acids}

Among the twenty compounds (nineteen phenolic acids and cinnamic acid) considered under the study, seven phenolic acids were determined in all the analyzed extracts from biomass cultivated under different lighting conditions: gallic, chlorogenic, $p$-hydroxybenzoic, protocatechuic, salicylic, syringic and vanillic acids.

The total amounts of these acids were also dependent on light quality and ranged from $23.54 \mathrm{mg} / 100 \mathrm{~g}$ DW to $46.64 \mathrm{mg} / 100 \mathrm{~g}$ DW. The highest overall amount of phenolic acids was obtained in extracts from the biomass maintained under blue light. A high, satisfactory total amount $(41.62 \mathrm{mg} / 100 \mathrm{~g} \mathrm{DW})$ was also obtained in darkness. Red light and far-red light proved to be less favourable for the accumulation of the studied metabolites (total contents reached $32.49 \mathrm{mg} / 100 \mathrm{~g}$ DW and $31.67 \mathrm{mg} / 100 \mathrm{~g}$ DW, respectively). The lowest total amount was obtained under UV-A irradiation. In extracts from the biomass cultivated under the control conditions (under white light), the total amount of the estimated free phenolic acids was $34.03 \mathrm{mg} / 100 \mathrm{~g} \mathrm{DW}$ (Table 2).

The amounts of the particular metabolites varied widely, depending on light quality, and ranged from 0.60 to $15.33 \mathrm{mg} / 100 \mathrm{~g} \mathrm{DW}$. The maximum amounts for most of the individual free phenolic acids were obtained in extracts from the biomass cultivated under blue light. The dominant compounds were: chlorogenic acid $(15.33 \mathrm{mg} / 100 \mathrm{~g} \mathrm{DW})$, protocatechuic acid $(13.11 \mathrm{mg} / 100 \mathrm{~g} \mathrm{DW})$ and gallic acid (6.27 mg/100 g DW) (Table 2).

\section{Discussion}

Our study demonstrated that different light spectra had different effects on biomass growth in in vitro cultures of $S$. chinensis and the accumulation of the secondary metabolites studied (Tables 1, 2). Both the growth and the appearance of the biomass were best obtained under blue light (Fig. 1). These maximum biomass increments were satisfactory-about 4.4-fold within 30-day growth cycles. These maximal increments were 1.15 times higher than under white light.

The study also demonstrated that blue light influences the best accumulation of the most important group of bioactive metabolites biosynthesized by $S$. chinensis cells-the dibenzocyclooctadiene lignans. The total amount of these compounds was 1.31 times higher than under white light conditions (the control). Also, the amounts for most of the individual compounds were higher in extracts from the biomass cultivated under blue light (Table 1). This study indicated the importance of lighting conditions on the accumulation of this group of lignans.

The stimulating properties of the type of light on the morphogenesis of in vitro cultures has been a known and often-studied phenomenon (Lin et al. 2011; Mengxi et al. 2011; Rodríguez-Sahagún et al. 2011; Sivanesan et al. 2011). Nevertheless, lighting conditions are among the less frequently tested conditions affecting the primary and secondary metabolism in plant cell cultures. The good growth observed under blue light may be related to the favourable impact of these wavelengths on nuclear and 


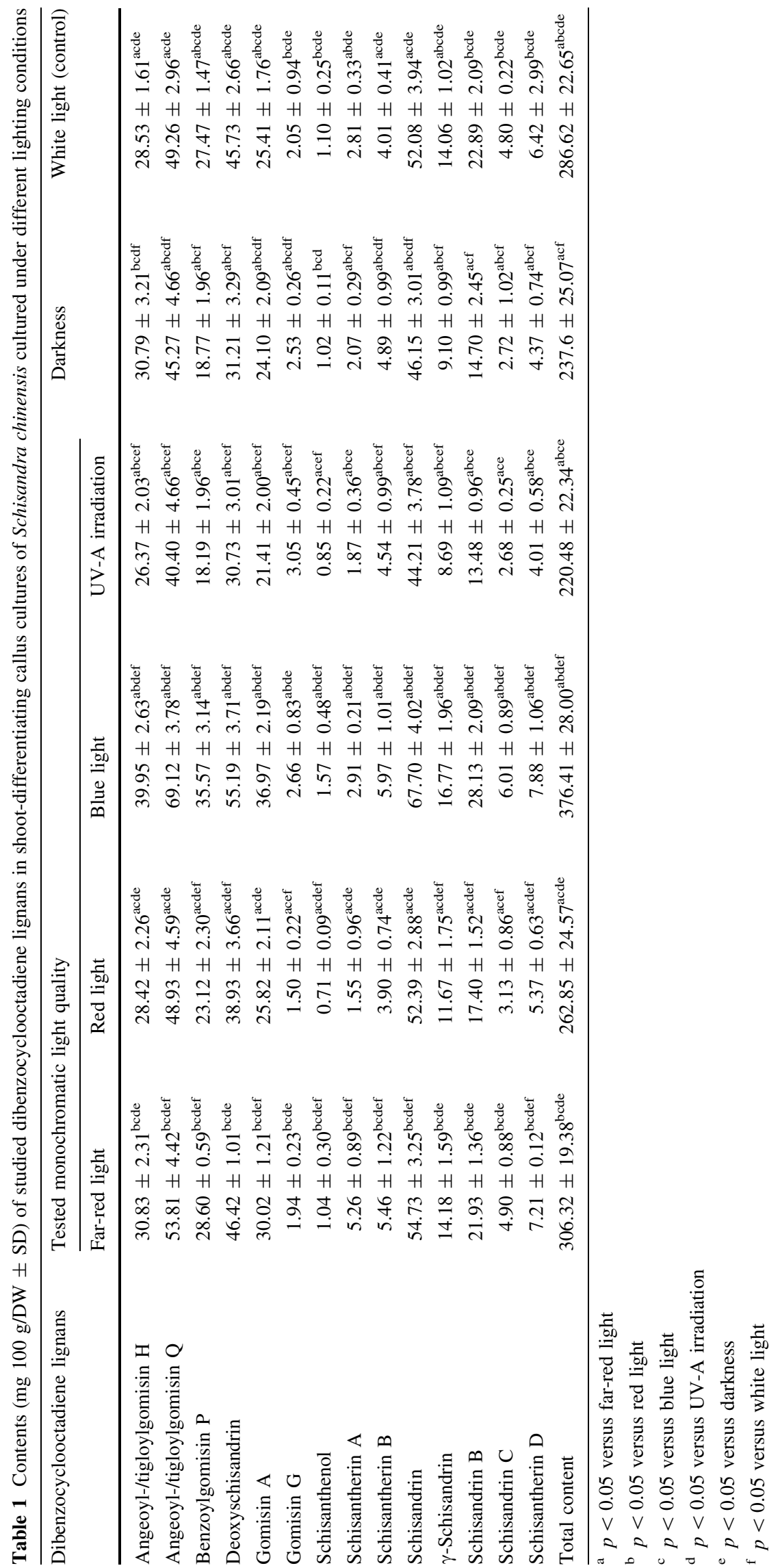


Table 2 Contents (mg 100 g/DW \pm SD) of studied free phenolic acids in shoot-differentiating callus cultures of Schisandra chinensis cultured under different lighting conditions

\begin{tabular}{|c|c|c|c|c|c|c|}
\hline \multirow[t]{2}{*}{ Phenolic acids } & \multicolumn{4}{|c|}{ Tested monochromatic light quality } & \multirow[t]{2}{*}{ Darkness } & \multirow[t]{2}{*}{ White light (control) } \\
\hline & Far-red light & Red light & Blue light & UV-A irradiation & & \\
\hline Chlorogenic acid & $8.43 \pm 2.03^{\mathrm{cf}}$ & $9.31 \pm 2.34^{\text {cde }}$ & $15.33 \pm 2.12^{\text {abdef }}$ & $5.63 \pm 0.97^{\text {bcef }}$ & $10.55 \pm 2.04^{\mathrm{acd}}$ & $11.93 \pm 1.81^{\text {acde }}$ \\
\hline Gallic acid & $3.61 \pm 1.02^{\mathrm{c}}$ & $3.75 \pm 1.07^{\mathrm{c}}$ & $6.27 \pm 0.94^{\mathrm{abdf}}$ & $4.39 \pm 1.03^{\mathrm{b}}$ & $4.75 \pm 0.47^{\mathrm{a}}$ & $3.91 \pm 0.32$ \\
\hline$p$-Hydroxybenzoic acid & $1.76 \pm 0.63$ & $1.82 \pm 0.48^{\mathrm{cd}}$ & $2.33 \pm 0.55^{\mathrm{d}}$ & $1.08 \pm 0.41^{\mathrm{ce}}$ & $2.31 \pm 0.31^{\mathrm{d}}$ & $1.49 \pm 0.47$ \\
\hline Protocatechuic acid & $10.45 \pm 3.03^{\mathrm{cd}}$ & $10.09 \pm 1.94^{\mathrm{f}}$ & $13.11 \pm 3.01^{\mathrm{abdef}}$ & $6.48 \pm 0.31^{\text {abcef }}$ & $10.45 \pm 1.87^{\mathrm{cd}}$ & $9.60 \pm 0.84^{\mathrm{ce}}$ \\
\hline Salicylic acid & $3.87 \pm 1.03^{\mathrm{e}}$ & $3.80 \pm 0.27^{\mathrm{e}}$ & $4.92 \pm 0.44^{\mathrm{cdf}}$ & $3.49 \pm 0.46^{\mathrm{ce}}$ & $9.49 \pm 1.99^{\mathrm{abcdf}}$ & $3.67 \pm 0.71^{\mathrm{d}}$ \\
\hline Syringic acid & $0.97 \pm 0.23$ & $1.04 \pm 0.43$ & $0.98 \pm 0.26^{\mathrm{d}}$ & $0.60 \pm 0.06^{\text {bce }}$ & $1.11 \pm 0.49$ & $0.82 \pm 0.32^{\mathrm{d}}$ \\
\hline Vanillic acid & $2.58 \pm 0.99^{\mathrm{c}}$ & $2.69 \pm 0.73$ & $3.64 \pm 0.33^{\mathrm{abdf}}$ & $1.87 \pm 0.30^{\text {bce }}$ & $2.96 \pm 0.60^{\mathrm{d}}$ & $2.62 \pm 0.55^{\mathrm{c}}$ \\
\hline Total content & $31.67 \pm 8.96^{\mathrm{cde}}$ & $32.49 \pm 7.26^{\mathrm{cde}}$ & $46.57 \pm 7.65^{\text {abdef }}$ & $23.54 \pm 3.54^{\text {abcef }}$ & $41.62 \pm 7.77^{\mathrm{abcdf}}$ & $34.03 \pm 5.02^{\mathrm{cdf}}$ \\
\hline \multicolumn{7}{|c|}{${ }^{\mathrm{a}} p<0.05$ versus far-red light } \\
\hline \multicolumn{7}{|c|}{${ }^{\mathrm{b}} p<0.05$ versus red light } \\
\hline \multicolumn{7}{|c|}{${ }^{\mathrm{c}} p<0.05$ versus blue light } \\
\hline \multicolumn{7}{|c|}{${ }^{\mathrm{d}} p<0.05$ versus UV-A irradiation } \\
\hline \multicolumn{7}{|c|}{ e $p<0.05$ versus darkness } \\
\hline \multicolumn{7}{|c|}{${ }^{\mathrm{f}} p<0.05$ versus white light } \\
\hline
\end{tabular}

plastid genomes in the evolution of plant cell chloroplasts (Richter and Wessel 1985). Moreover, some reported data indicate that changes in the spectral quality of illumination affect the composition of pigment-proteins, which are essential for the functioning of the photosynthetic apparatus (Topchiy et al. 2005). They may, therefore, contribute to greater growth because of higher photosynthetic efficiency under blue light, and, as a result, increase the production of secondary metabolites (Ramakrishna and Ravishankar 2011). Blue light has been found to promote the accumulation of, among others, anthocyanin glycosides in Haplopappus gracilis and Populus sp. (Ramawat and Mathur 2007) in vitro cultures. More recent experiments have also demonstrated this influence (Alvarenga et al. 2015; Kokotkiewicz et al. 2014; Szopa et al. 2012). Blue light also promotes the accumulation of hesperidin and some isoflavones in callus cultures of Cyclopia subternata (Kokotkiewicz et al. 2014), and of some volatile compounds in shoot cultures of Achillea millefolium (Alvarenga et al. 2015). Our studies of the accumulation of linear furanocoumarins in $R$. graveolens shoot cultures are another example (Szopa et al. 2012).

The experiments described here also proved the stimulating effect of light quality, especially the influence of blue light and darkness on the accumulation of free phenolic acids in the biomass of $S$. chinensis cultivated in vitro. The obtained total amounts of phenolic acids were respectively 1.37 and 1.22 times higher than in the biomass cultivated under the control conditions (white light). Also, the amounts of most of the individual free phenolic acids estimated in extracts from the biomass cultivated under blue light or in darkness were higher than under the control white light (Table 2).
Some experimental works from other research centres have demonstrated that the lack of light stimulates the biosynthesis of various classes of secondary metabolites, e.g. different groups of alkaloids and naphthoquinone pigments (Ramawat and Mathur 2007; Zhang et al. 2010). Our earlier studies had shown the same relationship for another group of secondary metabolites-linear furanocoumarins, where the lack of light favoured the accumulation of bergapten in Ammi majus callus cultures (Ekiert 1993), and also of isopimpinellin and xanthotoxin in $R$. graveolens ssp. divaricata shoot-differentiating callus cultures (Szopa et al. 2012).

Our present results confirm that the most advantageous lighting quality for the production of different groups of plant metabolites under in vitro conditions should be chosen empirically. A study on the impact of light quality on plant cultures in vitro is an important aspect of biotechnological investigations, mainly due to the fact that they are easy to implement under in vitro conditions. Based on extensive investigations, the blue light spectrum can be chosen as favouring biomass increments and also the production of dibenzocyclooctadiene lignans and free phenolic acids in shoot-differentiating callus cultures of S. chinensis.

Favourable lighting conditions (e.g. continuous blue light and/or darkness) can be applied to in vitro cultures without difficulty and the biomass cultured under such conditions can be proposed as a rich potential source of therapeutically valuable metabolites investigated in our study.

We propose $S$. chinensis cultures cultivated in vitro under blue light as a plausible source of some bioactive dibenzocyclooctadiene lignans-schisandrin, deoxyschisandrin and gomisin A, and phenolic acids-chlorogenic acid and protocatechuic acid. 
Acknowledgments The effect of wavelength was investigated in biotechnological laboratory of Department of Ornamental Plants, University of Agriculture in Kraków, Poland through the good offices of the Head of the Department dr hab. B. Pawłowska.

Open Access This article is distributed under the terms of the Creative Commons Attribution 4.0 International License (http://creative commons.org/licenses/by/4.0/), which permits unrestricted use, distribution, and reproduction in any medium, provided you give appropriate credit to the original author(s) and the source, provide a link to the Creative Commons license, and indicate if changes were made.

\section{References}

Alvarenga ICA, Pacheco FV, Ventorim F, Silva ST, Bertolucci SKV, Pinto JEB (2015) In vitro culture of Achillea millefolium L.: quality and intensity of light on growth and production of volatiles. Plant Cell Tiss Organ Cult 122:299-308

Chlopčikova Š, Psotová J, Miteková P, Soušek J, Lichnovský V, Šimánek V (2004) Chemoprotective effect of plant phenolics against anthracycline-induced toxicity on rat cardiomyocytes-caffeic, chlorogenic and rosmarinic acids. Phytother Res 18:408-413

Ekiert H (1993) Ammi majus L. (Bishop's weed): in vitro culture and the production of coumarin compounds. In: Bajaj YPS (ed) Biotechnology in agriculture and forestry, medicinal and aromatic plants, vol IV. Springer, Berlin, pp 1-17

Ellnain-Wojtaszek M, Zgórka G (1999) High-performance liquid chromatography and thin-layer chromatography of phenolic acids from Ginkgo biloba $\mathrm{L}$. leaves collected within vegetative period. J Liq Chromatogr Relat Technol 22:1457-1471

European Directorate for the Quality of Medicines (2008) European pharmacopoeia, vol 6.3. Schisandra fruit, Strasburg

Hancke JL, Burgos RA, Ahumada F (1999) Schisandra chinensis (Turcz.) Baill. Fitoterapia 70:451-471

Khadem S, Marles RJ (2010) Monocyclic phenolic acids; hydroxyand polyhydroxybenzoic acids: occurrence and recent bioactivity studies. Molecules 15:7985-8005

Kokotkiewicz A, Bucinski A, Luczkiewicz M (2014) Light and temperature conditions affect bioflavonoid accumulation in callus cultures of Cyclopia subternata Vogel (honeybush). Plant Cell Tiss Organ Cult 118:589-593

Krewzaler F, Hahlbrock K (1973) Flavonoid glycosides from illuminated cell suspension cultures of Petroselinum hortense. Phytochemistry 12:1149-1152

Lin Y, Li J, Li B, He T, Chun Z (2011) Effects of light quality on growth and development of protocorm-like bodies of Dendrobium officinale in vitro. Plant Cell Tiss Organ Cult 105:329-335

Mengxi L, Zhigang X, Yang Y, Yijie F (2011) Effects of different spectral lights on Oncidium PLBs induction, proliferation, and plant regeneration. Plant Cell Tiss Organ Cult 106:1-10

Murashige T, Skoog F (1962) A revised medium for rapid growth and bioassays with tobacco tissue cultures. Physiol Plant 15:473-497

Ramakrishna A, Ravishankar GA (2011) Influence of abiotic stress signals on secondary metabolites in plants. Plant Signal Behav 6:1720-1731

Ramawat KG, Mathur M (2007) Factors affecting the production of secondary metabolites. In: Ramawat KG, Merillon JM (eds) Biotechnology: secondary metabolites. Plants and microbes. Science Publ Inc., Enfield, pp 59-102

Richter G, Wessel K (1985) Red light inhibits blue-induced chloroplast development in cultured plant cells at the mRNA level. Plant Mol Biol 5:175-182

Rodríguez-Sahagún A, Acevedo-Hernández G, Rodríguez-Domínguez JM, Rodríguez-Garay B, Cervantes-Martínez J,
Castellanos-Hernández OA (2011) Effect of light quality and culture medium on somatic embryogenesis of Agave tequilana Weber var. Azul. Plant Cell Tiss Organ Cult 104:271-275

Shin SK, Murthy HN, Heo JW, Paek KY (2004) Induction of betalain pigmentation in hairy roots of red beet under different radiation sources. Biol Plant 47:149-152

Sivanesan I, Lim MY, Jeong BR (2011) Somatic embryogenesis and plant regeneration from leaf and petiole explants of Campanula punctata Lam. var. rubriflora Makino. Plant Cell Tiss Organ Cult 107:365-369

Szopa A, Ekiert H (2011) Lignans in Schisandra chinensis in vitro cultures. Pharmazie 66:633-634

Szopa A, Ekiert H (2012) In vitro cultures of Schisandra chinensis (Turcz.) Baill. (Chinese magnolia vine) - a potential biotechnological rich source of therapeutically important phenolic acids. Appl Biochem Biotechnol 166:1941-1948

Szopa A, Ekiert H (2013) Production of deoxyschizandrin and $\gamma$ schizandrin in shoot-differentiating and undifferentiating callus cultures of Schisandra chinensis (Turcz.) Baill. (Chinese magnolia vine). J Biotechnol 165:209-213

Szopa A, Ekiert H (2014) Schisandra chinensis (Turcz.) Baill. (Chinese magnolia vine) in vitro cultures. In: Govil JN (ed) Recent progress in medicinal plants. Biotechnology and genetic engineering II, vol 39. Studium Press LLC, Houston, pp 405-434

Szopa A, Ekiert H (2015) Production of schisantherin A and gomisin G in in vitro cultures of Schisanda chinensis. Phytochem Lett 11:440-444

Szopa A, Ekiert H, Szewczyk A, Fugas E (2012) The production of therapeutically important phenolic acids and furanocoumarins in in vitro cultures of Ruta graveolens and Ruta graveolens ssp. divaricata cultured under different light conditions. Plant Cell Tiss Organ Cult 110:329-336

Szopa A, Ekiert H, Kisiel W, Szewczyk A (2015) Isolation of three dibenzocyclooctadiene lignans from in vitro cultures of Schisandra chinensis (Turcz.) Baill.—-the first report. Pharmazie 70:337-339

Szopa A, Ekiert R, Ekiert H (2016a) Current knowledge of Schisandra chinensis (Turcz.) Baill. (Chinese magnolia vine) as a medicinal plant species: a review on the bioactive components, pharmacological properties, analytical and biotechnological studies. Phytochem Rev. doi:10.1007/s11101-016-9470-4

Szopa A, Kokotkiewicz A, Marzec-Wróblewska U, Bucinski A, Luczkiewicz M, Ekiert H (2016b) Accumulation of dibenzocyclooctadiene lignans in agar cultures and in stationary and agitated liquid cultures of Schisandra chinensis (Turcz.) Baill. Appl Microbiol Biotechnol 100:3965-3977

Topchiy NM, Sytnik SK, Syvash OO, Zolotareva OK (2005) The effect of additional red irradiation on the photosynthetic apparatus of Pisum sativum. Photosynthetica 43:451-456

US Pharmacopeial Convention (1999) United States PharmacopeiaNational Formulary. Schisandra chinensis fruit. Pharmaceutical Compounding-Sterile Products, Rockville

World Health Organization (2007) WHO monographs on selected medicinal plants, vol. 3. Fructus Schisandrae. Geneva, pp 296-313

Yu KW, Murthy HN, Hahn EJ, Paek KY (2005) Ginsenoside production by hairy root cultures of Panax ginseng: influence of temperature and light quality. Biochem Eng J 23:53-56

Zhang H, Zhang G, Zhu Z, Zhao L, Fei Y, Jing J, Chai Y (2009) Determination of six lignans in Schisandra chinensis (Turcz.) Baill. fruits and related Chinese multiherb remedies by HPLC. Food Chem 115:735-739

Zhang WJ, Su J, Tan MY, Liu GL, Pang YJ, Shen HG, Qi JL, Yang Y (2010) Expression analysis of shikonin-biosynthetic genes in response to M9 medium and light in Lithospermum erythrorhizon cell cultures. Plant Cell Tiss Organ Cult 101:135-142

Zhong JJ, Seki T, Kinoshita S, Yoshida T (1991) Effect of light irradiation on anthocyanin production by suspended cultures of Perilla frutescens. Biotechnol Bioeng 38:653-658 